\title{
BMJ Open Mixed-methods study protocol: do national reporting and learning system medication incidents in palliative care reflect patient and carer concerns about medication management and safety?
}

\author{
Sarah Yardley (1) , ${ }^{1,2}$ Sally-Anne Francis (1) , ${ }^{1}$ Antony Chuter, ${ }^{3}$ Stuart Hellard, ${ }^{4}$ \\ Julia Abernethy, ${ }^{5}$ A Carson-Stevens ${ }^{4}$
}

To cite: Yardley S, Francis SA, Chuter A, et al. Mixedmethods study protocol: do national reporting and learning system medication incidents in palliative care reflect patient and carer concerns about medication management and safety? BMJ Open 2021;11:e048696. doi:10.1136/ bmjopen-2021-048696

- Prepublication history and additional supplemental material for this paper are available online. To view these files, please visit the journal online (http://dx.doi.org/10.1136/ bmjopen-2021-048696).

Received 04 January 2021 Accepted 13 August 2021

Check for updates

(C) Author(s) (or their employer(s)) 2021. Re-use permitted under CC BY-NC. No commercial re-use. See rights and permissions. Published by BMJ.

For numbered affiliations see end of article.

Correspondence to

Dr A Carson-Stevens;

carson-stevensap@cardiff.ac.uk

\section{ABSTRACT}

Introduction Approximately $20 \%$ of serious safety incidents involving palliative patients relate to medication. These are disproportionately reported when patients are in their usual residence when compared with hospital or hospice. While patient safety incident reporting systems can support professional learning, it is unclear whether these reports encompass patient and carer concerns with palliative medications or interpersonal safety.

Aim To explore and compare perceptions of (un)safe palliative medication management from patient, carer and professional perspectives in community, hospital and hospice settings.

Methods and analysis We will use an innovative mixed-methods study design combining systematic review searching techniques with cross-sectional quantitative descriptive analysis and interpretative qualitative metasynthesis to integrate three elements: (1) Scoping review: multiple database searches for empirical studies and first-hand experiences in English (no other restrictions) to establish how patients and informal carers conceptualise safety in palliative medication management. (2)Medication incidents from the England and Wales National Reporting and Learning System: identifying and characterising reports to understand professional perspectives on suboptimal palliative medication management. (3) Comparison of 1 and 2: contextualising with stakeholder perspectives.

Patient and public involvement Our team includes a funded patient and public involvement (PPI) collaborator, with experience of promoting patient-centred approaches in patient safety research. Funded discussion and dissemination events with PPI and healthcare (clinical and policy) professionals are planned.

Ethics and dissemination Prospective ethical approval granted: Cardiff University School of Medicine Research Ethics Committee (Ref 19/28). Our study will synthesise multivoiced constructions of patient safety in palliative care to identify implications for professional learning and actions that are relevant across health and social care. It will also identify changing or escalating patterns in palliative medication incidents due to the COVID-19 pandemic. Peer-reviewed publications, academic
Strengths and limitations of this study

- There is a moral imperative to learn from data about potentially unsafe healthcare, as advocated by WHO.

- This study addresses patient and carer concerns about medication management and safety in palliative care, which is complex, poorly defined and under-researched.

- This is an innovative mixed-method study combining systematic review searching techniques with cross-sectional quantitative descriptive analysis and interpretative qualitative meta-syntheses.

- When data are drawn from existing published literature and from a national incident reporting database thereby focusing on what people choose to say or report, it remains possible that some specific elements may be under-represented or over-represented.

- The addition of an 'expert by experience' voice into the study design is helping to ensure the research remains focused on patient needs and experiences.

presentations, plain English summaries, press releases and social media will be used to disseminate to the public, researchers, clinicians and policy-makers.

\section{PLAIN ENGLISH ABSTRACT (WRITTEN WITH OUR PPI COLLABORATOR)}

We know that people only have high quality healthcare experiences if they feel safe. This relies on their concerns being heard, and decisions about their care being made with them. It is more than just being technically safe, but even that is not always achieved. The National Reporting and Learning System (NRLS) is a system for people working in the National Health Service (NHS) to report safety incidents. Medication safety incidents account for more than $10 \%$ of NRLS reports. In Supportive, Palliative and End-of-Life Care, medicines are commonly used to help with 
symptoms such as pain, nausea, vomiting, chest secretions and restlessness. Patient and carers views on good medicines safety in palliative care have not been compared with what people working in healthcare report to the NRLS. In this study we are going to compare patient and carer views (taken from published research) with a sample of NRLS incidents. This will help us to better understand what is meaningful to patients in palliative care and what is needed to ensure medication and patient safety works. We will look for gaps that can be addressed by targeting support for learning and change. The way we are doing this study (methods) has not been done before and so we will also contribute to new ways for doing safety and healthcare improvement research.

\section{INTRODUCTION}

The third WHO Global Patient Safety Challenge 'Medication Without Harm' is an initiative seeking to halve severe avoidable harm due to medication-related patient safety incidents by 2022. ${ }^{1}$ In England, such incidents account for more than $10 \%$ of the total National Reporting and Learning System (NRLS) patient safety incident reports (eg, Most up to date version referenced. This is updated every 6 months. Published data are only ever for England as data collected on behalf of Wales is not part of National Health Service (NHS) England's remit for publication), ${ }^{2}$ while approximately $20 \%$ of serious incident reports involving palliative patients in England and Wales in the Strategic Executive Information Management System relate to prescribing or prescription medication management (eg, In the NHS distinctions are made between patient safety incidents (any unintended or unexpected incident which could have, or did, lead to harm for one or more patients receiving healthcare) and serious incidents (which can extend beyond incidents which affect patients directly and include incidents which may indirectly impact patient safety or an organisation's ability to deliver ongoing healthcare) although the two categories are not mutually exclusive). ${ }^{23}$ Prescribing and medication are well-acknowledged sources of risk-prone healthcare activity. ${ }^{4-11}$ Yet, despite an accumulation of evidence regarding the size of the problem nationally and internationally, ${ }^{3}{ }^{12-21}$ the circumstances, actions or influences that play a part in their origin or development of such incidents ${ }^{22}$ ('contributing factors') are not well understood. Practice etiquette ${ }^{23}$ shapes prescribing far more than protocols, guidelines and scientific evidence, even when available and often evidence is lacking for normative practice in palliative care. ${ }^{24}$

'Safety' describes the notion of reducing the risk of unnecessary harm to an acceptable minimum, where acceptable minimum refers to the collective notions of given current professional knowledge, resources available and the context in which care is delivered weighed against the risk of non-treatment, treatment or other intervention. ${ }^{22}$ Viewed in these terms, healthcare is an inherently risky and potentially unsafe endeavour. Both acts and omissions have high stakes with potentially serious unintended consequences. Agreement that a person is receiving safe care depends on multiple interconnected system components that are mediated through dynamic human interactions and interventions. ${ }^{25}$ Recognising this within healthcare, patient safety is defined as 'the avoidance of unintended or unexpected harm to people during the provision of healthcare'. ${ }^{26}$

Patients receiving palliative care are vulnerable to safety incidents in community (ie, home or usual residence), hospital and hospice settings yet often these patients are considered differently to other populations when risks are assessed. ${ }^{327}$ Medication errors are disproportionately reported when patients are being cared for in their own homes. ${ }^{3}$ Expectations of primary and acute care professionals to prescribe for symptom control contrast with reported hindrances of lack of time, confidence and skills. ${ }^{188-30}$ The predominant strategy for palliative care coordination relies on informal personal relationships between professionals, shared norms, values and mutual trust. ${ }^{31}$ Under-resourcing and poor service coordination contribute to a situation where the healthcare system is perfectly designed for patient safety incidents to occur. ${ }^{29}$ This makes it unlikely that patients will universally receive the 'right care at the right time' from the right person despite only 'one chance to get it right'. 3233

Assumptions that safety, or care quality, are lesser concerns in the context of progressive life-limiting illness are not justifiable. Safety remains important to patients and their families in the context of lifelimiting illness. ${ }^{34}$ Patients and carers experience harm as an unfolding series of negative events creating lack of interpersonal safety rather than simply being limited to technical-clinical care. ${ }^{34}{ }^{35}$ Actual harm is compounded by being 'inextricably linked with feeling unsafe'. ${ }^{34}$ There is, however, a surprising paucity of research in this area. ${ }^{18}$

Patient safety incident reporting systems, such as the NRLS, are intended to support the generation of learning from incidents and near misses to inform strategies to reduce harm to future patients. We do not know if patient safety incidents reported, predominantly by healthcare professionals, encompass or resonate with patient and carer concerns about 'safe use of medications' in palliative care ${ }^{36}$ or whether reported incidents can be reviewed for learning taking into account the broader context of interpersonal safety. ${ }^{34}$ Through analysis of multiple perspectives from routine patient safety data sources, we anticipate identifying patient-centred priorities to inform targeted quality improvement interventions.

This protocol has been reviewed and revised in the light of the COVID-19 pandemic. Evidence is now emerging of a rapidly evolving model of clinical practice occurring in palliative care, with new ways of shared working between specialists and generalists and greater use of remote monitoring. ${ }^{37} 38$ In addition, both numbers of deaths and place of death is undergoing rapid change, with a massive escalation in deaths everywhere except in hospices. ${ }^{39}$ There is a greater dependency on families 


\section{Box 1 Perspectives for synthesis}

1. Front-line healthcare professionals ${ }^{1}$ : identified through reporting of clinical incidents. ${ }^{1}$

2. Patient/informal carer and general public constructions of medication management and safety: identified through a scoping review.

and friends with respect to medication management and in some cases administration, including controlled and injectable medicines. Anticipatory prescribing guidance and practice changes have occurred with alterations in route, choice of drug, quantities, doses and dose ranges all reported. ${ }^{38} 40$ Clinicians are also exploring alternative uses of medications and alternative routes of administration, with which many professionals outside of specialist palliative care would be less familiar. ${ }^{41}$ Given the expectation of repeated waves of COVID-19, 'the marathon not a sprint', and potential for practice evolutions to have impact long beyond the pandemic, it is of critical importance we gain a better understanding of current safe and unsafe palliative medication management in all forms and settings. This study will allow us to identify early any changing or escalating patterns in medication safety incidents due to the pandemic.

\section{Aim}

The aim of this study is to explore and compare perceptions of (un) safe palliative medication management from patient, carer and professional perspectives in community, hospital and hospice settings (see box 1).

The objectives are to:

1. Conceptualise how patients, carers and others important to themiii make sense of safety in palliative care; specifically identifying their concerns about medication management in palliative care.

2. Identify, characterise and analyse professional perspectives on suboptimal palliative care in front-line healthcare practice in relation to medication-related patient safety incidents.

3. Compare and contrast patients' and carers' perspectives on medication management with those of healthcare professionals, to identify areas for further research and quality improvement activities.

4. Identify any early signs of changing or escalating patterns in palliative medication safety incidents in the context of COVID-19.

\section{Patient and public involvement methods}

This study addresses issues identified by the James Lind Alliance Palliative and End-of-Life Care Priority Setting Partnership which included public, patient and carer consultation. ${ }^{42}$ All members of the research team and patient and public involvement (PPI) groups will be required to ensure everyone respects and values the contribution of all collaborating in our research. ${ }^{43}$ Integral to the study is the belief that it is only by everyone working together, can we identify priority targets for meaningful change to achieve effective prescribing and medication management.

Our core study team includes a PPI collaborator. The addition of a PPI 'expert by experience' voice into the study design and processes is helping to ensure the research remains focused on patient needs and experiences as well as clinical practice. He has been involved from the inception of the study and throughout its design, contributing to the development of this protocol alongside the academic and professional team members. He will be fully engaged in study team meetings during the analytical and interpretative stages as well as contributing to the outputs of the work. ${ }^{44}$

The PPI collaborator has provided a specific advice on the methods for further PPI and will be an additional contact for PPI group members to discuss any aspect of the study, including ethical concerns. ${ }^{45}$ Two PPI group events will be held ( $n=10$, either face to face or remotely, pending pandemic restrictions). People with experience of palliative care either as patients or carers will be invited to attend and discuss the emerging study findings to help focus the work on issues and priorities most important to them and others like them. Attendees will be invited to co-produce conference submissions and academic publications, with flexible approaches to involvement in order to support inclusivity. ${ }^{46}$ Communications will be welcomed between all involved in the research and conducted as far as possible in Plain English, with explanations of any necessary technical terms. ${ }^{47} 48$ This will broaden and strengthen the PPI perspective in the research.

\section{Conceptual model}

This study is theoretically aligned to sociocultural constructionist and interpretivist approaches, ${ }^{49-51}$ accepting that people act based on how they have made sense of lived experience, including interactions with other people within a system, and that prior learning impacts on future experience and capacity for further learning. We will bring together analysis of patient and informal carer perspectives and professionals reports of potential or actual harms. In doing so, we will be able to identify opportunities where shared understanding and priorities can be capitalised and where differences of perspective may bring about concerns, conflict or communication breakdowns in patient-centred care. This is an important starting point for developing a holistic view of patient safety within palliative care given its reliance on informal carers as well as generalist and specialist professional providers.

\section{METHODS AND ANALYSIS}

The study will draw on an innovative mixed-method combination of systematic review searching techniques with published literature and cross-sectional quantitative descriptive analysis and interpretative qualitative metasyntheses of incident data from the England and Wales NRLS, contextualised in stakeholder perspectives. ${ }^{52} 53$ 


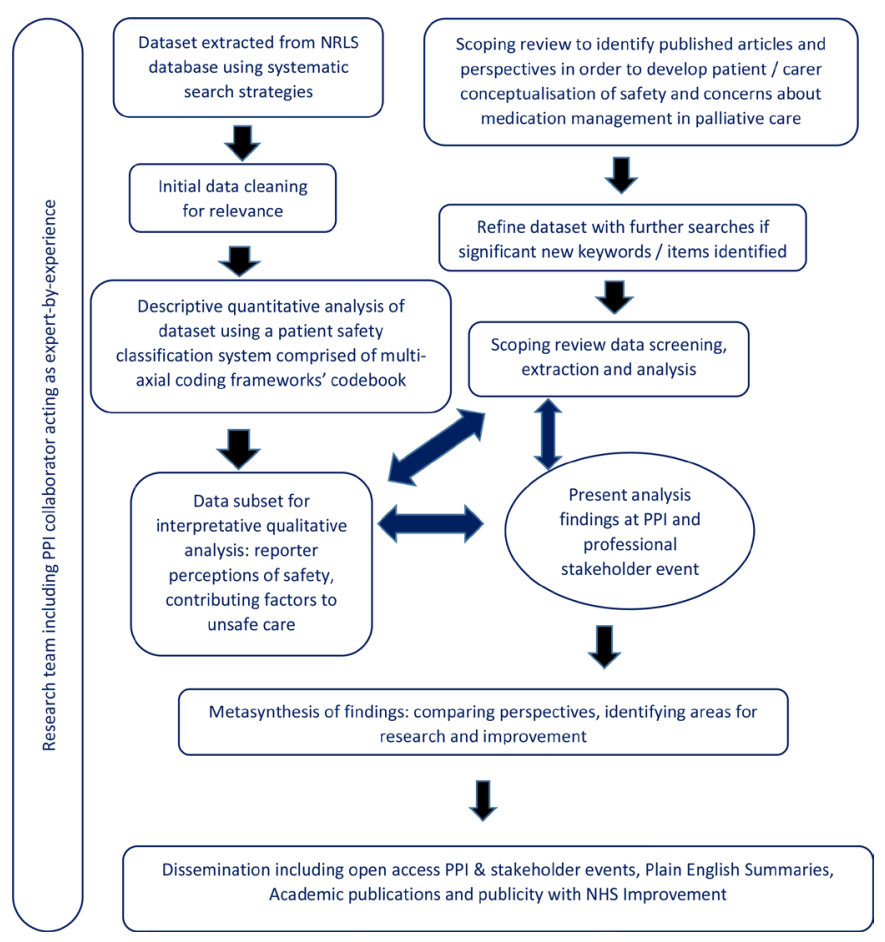

Figure 1 Study flow diagram. NHS, National Health Service; NRLS, National Reporting and Learning System; PPI, patient and public involvement.

An overview of the study, showing order and timing of data analysis and integration is given in figure 1. Details of the systematic search strategy for the scoping review are given in tables 1-4 and Boxes 2 and 3. Details of the systematic search strategy for the NRLS database are given in figure 2 and tables 5 and 6 . In line with the study methodology, iterative changes will be documented if made as the work progresses.

\section{Study design}

\section{Scoping review}

A scoping review provides 'knowledge synthesis that addresses an exploratory research question aimed at mapping key concepts, types of evidence, and gaps in research related to a defined area or field by systematically searching, selecting, and synthesising existing knowledge ${ }^{54-56}$ We will use standard systematic search techniques (aligned to PRISMA for Scoping Reviews (PRISMA ScR)) to identify existing literature, drawing on principles from the Joanna Briggs Institute $\mathrm{e}^{57}$ and interpretative approaches to data synthesis. ${ }^{58-60} \mathrm{~A}$ minimum of two reviewers will be involved at each stage of the work. The scoping review protocol has been peer reviewed by an academic librarian. The planned steps in our search, inclusion and exclusion criteria are provided in box 2 .

The search thread was created using 'Population, Concept, Context' (PCC) as per table 1 with PCC combined as ((Population: patients; informal carers) AND (Concept: safety OR Concept: medication management) AND Context: palliative care)). Table 2 shows our first translation of this thread into search terms for each concept. We used these as a starting point for preliminary searches to develop our initial limited search, thereby ensuring the final search would be appropriately sensitive and specific.

After testing V.1 of our search terms we refined this to create the initial limited Medline search strategy that we will use (see online supplemental file 1).

In developing this, we drew on Brooks et $a l^{61}$ to refine the chosen population search terms. We also reviewed Collier $e t a \vec{l}^{4}$ for keywords (all included). During this process, some search terms were added/removed (see box 2).

Once the initial limited search had been refined in Medline we translated terms suitable for Embase. Initially running the refined Medline version with simple translation of MESH to SH terms produced a vast and unmanageable number of records. We, therefore, tested focusing (rather than exploding) and limiting keywords to titles/ abstracts in either the safety search concept or the population search. Table 3 shows the final search strategy using focused searches in Embase.

Overall, this has given us an estimated total of $\mathrm{N}=9995$ records to screen for the initial limited search.

\section{Quality assessment}

As we want to scope the available evidence, exclusions will not be made on the basis of quality. Quality assessment will, instead, be conducted using an adapted 5-point strength score $^{58}$ with each published manuscript being independently scored by two members of the research team and where the scores differ the lower score being accepted. Scores will be presented alongside the synthesis.

This approach allows us to draw on the framework of principles set out in Gough's Weight of Evidence ${ }^{62}$; making separate judgements on different types of data from different study criteria before combining these to make a global judgement of the contribution of a particular piece of evidence to answering our scoping review research questions. In doing so, assessment will be made of the coherence and integrity of each piece of evidence in their own terms, that is, using the 'generally accepted criteria for evaluating the quality of this type of evidence by those who generally use and produce it'. ${ }^{62}$ We will also consider how appropriate the methods and focus of each piece of evidence are for answering our questions. When possible we will use appropriate checklists to support our assessment, for example, Equator guidelines. ${ }^{63} \mathrm{~A}$ summary of our global quality assessment scoring system is in table 4 .

\section{Synthesis}

Given the nature of our research questions, the necessity for first person data from patients and informal carers, and from our knowledge of the field, we anticipate most inclusions will be qualitative, mixed-methods or descriptive quantitative data. We, therefore, anticipate qualitative metasynthesis will be the best method for our analysis and synthesis. This will be achieved by conducting a thematic analysis of first, second and third order data, and drawing 


\begin{tabular}{|c|c|c|}
\hline Criteria & $\begin{array}{l}\text { Search strands (see next } \\
\text { table for details) }\end{array}$ & Definitions \\
\hline Concept 1 & Safety & $\begin{array}{l}\text { Patient safety is the reduction of risk of unnecessary harm associated with healthcare to an acceptable } \\
\text { minimum. An acceptable minimum refers to the collective notions of given current knowledge, resources } \\
\text { available and the context in which care was delivered weighed against the risk of non-treatment or other } \\
\text { treatment. }^{22}\end{array}$ \\
\hline Concept 2 & Medication use/management & $\begin{array}{l}\text { 'Getting the right medication to the right person at the right time'. Encompassing the whole multi-step task } \\
\text { of: } \\
\text { Decision making/starting a medication. } \\
\text { Prescribing/taking/adding a medication. } \\
\text { Monitoring and supply/reviewing a medication. } \\
\text { Administration. } \\
\text { Stopping medications. } \\
\text { Moving across healthcare contexts. }{ }^{7475}\end{array}$ \\
\hline Context & $\begin{array}{l}\text { Last phase of life } \\
\text { palliative care } \\
\text { Of any type (eg, } \\
\text { specialist or generalist). } \\
\text { In any location. }\end{array}$ & $\begin{array}{l}\text { Last phase of life defined as having potentially life-limiting irreversible or progressive condition requiring } \\
\text { general or specialist palliative care for symptom control, social, psychological and/or spiritual support. Given } \\
\text { the challenges of prognostication, and tendency for this to be overestimated we have chosen not to include } \\
\text { a time frame in this definition. } \\
\text { 'Palliative care is an approach that improves the quality of life of patients and their families facing the } \\
\text { problem associated with life-threatening illness, through the prevention and relief of suffering by means } \\
\text { of early identification and impeccable assessment and treatment of pain and other problems, physical, } \\
\text { psychosocial and spiritual. Palliative care: } \\
\text { Provides relief from pain and other distressing symptoms. } \\
\text { Affirms life and regards dying as a normal process. } \\
\text { Intends neither to hasten or postpone death. } \\
\text { Integrates the psychological and spiritual aspects of patient care. } \\
\text { Offers a support system to help patients live as actively as possible until death. } \\
\text { Offers a support system to help the family cope during the patients illness and in their own bereavement. } \\
\text { Uses a team approach to address the needs of patients and their families, including bereavement } \\
\text { counselling, if indicated. } \\
\text { Will enhance quality of life, and may also positively influence the course of illness. } \\
\text { Is applicable early in the course of illness, in conjunction with other therapies that are intended to } \\
\text { prolong life, such as chemotherapy or radiation therapy, and includes those investigations needed to } \\
\text { better understand and manage distressing clinical complications.' https://www.who.int/cancer/palliative/ } \\
\text { definition/en/ }\end{array}$ \\
\hline
\end{tabular}

on meta-ethnographic methods to express the final synthesis. ${ }^{59}$ First order data refers to empirical data (eg, participant verbatim quotations) within published works, second order is the interpretation of the original study research team and third order is the interpretation of the synthesis team (ie, this study team). In addition, for all studies we will chart: authors; year of publication; origin of study; aims/purpose; population and sample size; methodology and methods; summary of key findings related to our scoping review questions and if applicable, intervention details, outcomes. Data extraction, analysis and synthesis will be completed as a team with independent coding of themes by at least two researchers, and cross-checking of all aspects of the process between team members.

\section{NRLS incident report dataset}

Context and database population

The NRLS is a central database set up to facilitate learning from incidents occurring within the NHS in England and Wales. Reporting is voluntary and encouraged for any unintended or unexpected incident that resulted in or could have resulted in harm to one or more patients receiving state funded care. ${ }^{64}$ The NRLS can also be used by NHS trusts as a mandatory reporting route for certain deaths and other incidents to fulfil the regulatory requirements made of them by the Care Quality Commission. Approximately 150000-200000 incidents are reported per month (more than 2 million per year) providing descriptive categorical information (incident details including reported type and location, patient demographics and reporter-rated severity of harm) and free-text reporter or organisational perspectives on what happened, perceived contributing factors and plans to minimise risk of reoccurrence.

\section{Sampling to generate the study population}

Our population of interest is all patients receiving medication for palliative purposes involved in a patient safety incident which has been reported to the NRLS. It is not limited to specialist palliative care settings or teams. 
Table 2 Search strands tested (V.1)

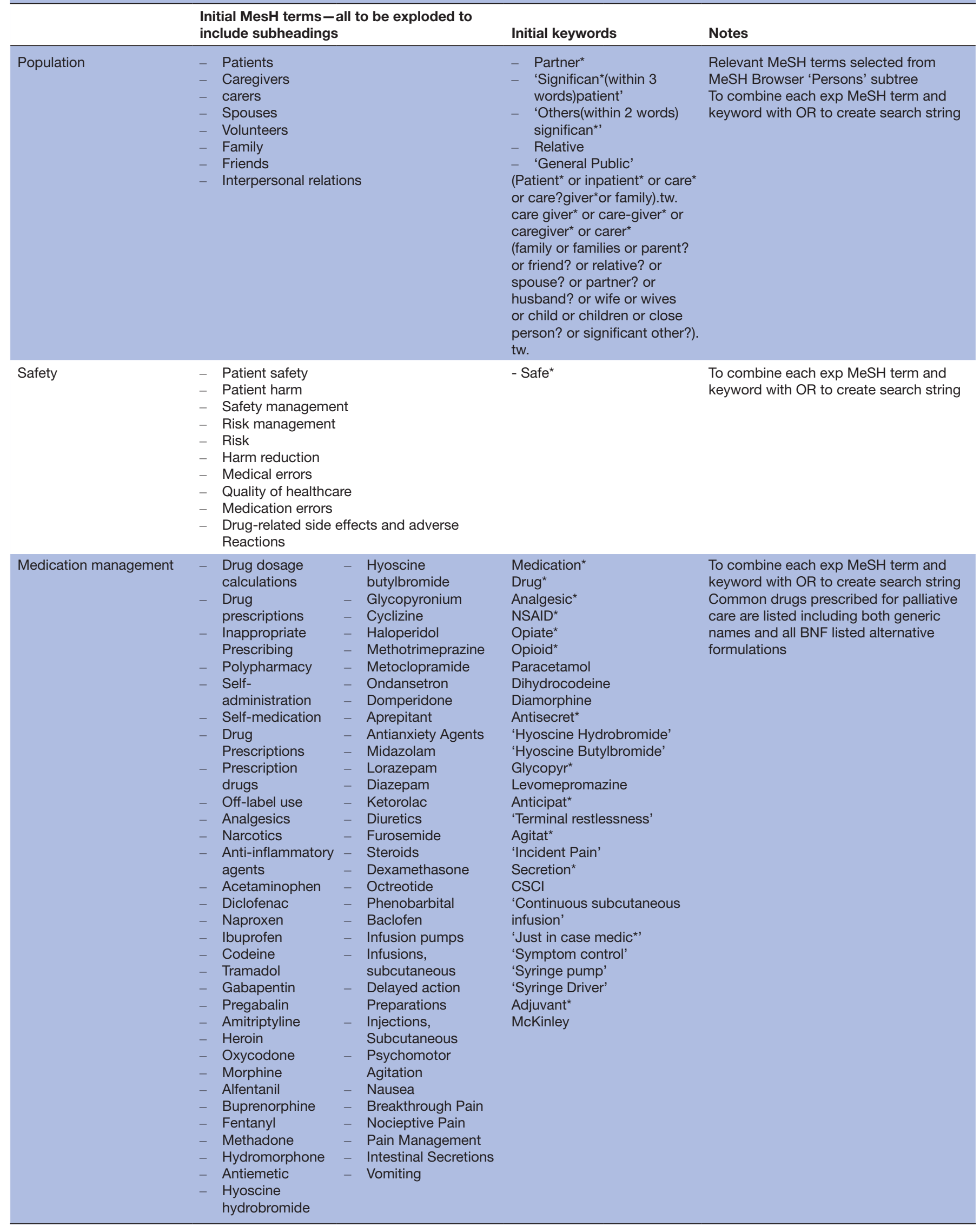

Continued 
Table 2 Continued

\begin{tabular}{|c|c|c|c|c|}
\hline \multirow[b]{2}{*}{ Palliative care } & \multirow{2}{*}{$\begin{array}{l}\text { Initial MesH terms - all to be exploded to } \\
\text { include subheadings } \\
\text { Terminally III } \\
\text { Terminal care } \\
\text { Palliative care } \\
\text { Hospice and palliative care nursing } \\
\text { Hospice care } \\
\text { Supportive care } \\
\text { Palliative medicine } \\
\text { Advance* care planning } \\
\text { Resuscitation orders }\end{array}$} & \multicolumn{2}{|c|}{ Initial keywords } & Notes \\
\hline & & $\begin{array}{l}\text { 'Last phase } \\
\text { of life' } \\
\text { 'Last days of } \\
\text { life' } \\
\text { 'Care of the } \\
\text { dying' } \\
\text { 'Liverpool } \\
\text { Care Pathway' } \\
\text { 'Electronic } \\
\text { Palliative Care } \\
\text { Co-ordination } \\
\text { Systems' } \\
\text { 'End of life' } \\
\text { End-of-life } \\
\text { Dying } \\
\text { 'Expected } \\
\text { death' } \\
\text { 'Inappropriate } \\
\text { admission' } \\
\text { 'Karnovsky } \\
\text { score' } \\
\text { 'living will' } \\
\text { 'Marie Curie' } \\
\text { 'Macmillan' } \\
\text { 'Phase of } \\
\text { illness' } \\
\text { 'preferred } \\
\text { place of care' }\end{array}$ & $\begin{array}{l}\text { 'unplanned } \\
\text { admission' } \\
\text { Palliat* } \\
\text { DNACPR } \\
\text { PPD } \\
\text { PPC } \\
\text { PPD } \\
\text { EPACCS } \\
\text { ACP } \\
\text { ADRT } \\
\text { ('last year of } \\
\text { life' or LYOL } \\
\text { or 'end of life' } \\
\text { or 'end of } \\
\text { their lives' or } \\
\text { 'last } 6 \text { months } \\
\text { of life' or 'last } \\
6 \text { months of } \\
\text { life') }\end{array}$ & $\begin{array}{l}\text { To combine each exp MeSH term and } \\
\text { keyword with OR to create search string }\end{array}$ \\
\hline
\end{tabular}

A study-specific search strategy using systematic review principles has been designed drawing on our collective expertise in incident reporting, informatics, pharmacology, clinical practice, lived experience and

\begin{tabular}{|c|c|}
\hline \multicolumn{2}{|l|}{$\begin{array}{l}\text { Search 1: Combine population AND (safety or medication } \\
\text { management) AND Palliative care }\end{array}$} \\
\hline V.1 for Embase & 506622 \\
\hline Revised Medline run direct in Embase & 53008 \\
\hline Revised Medline with Embase thesaurus & 38347 \\
\hline $\begin{array}{l}\text { Final SEARCH } 1 \text { with focused 'safety' strand (remainder } \\
\text { of search as before) }\end{array}$ & 1492 \\
\hline \multicolumn{2}{|l|}{ Duplicates: $\mathrm{N}=375$} \\
\hline Ready for title and abstract screening & 1117 \\
\hline \multicolumn{2}{|c|}{$\begin{array}{l}\text { Search 2: Combine Population AND (safety or medication management) } \\
\text { AND Palliative care }\end{array}$} \\
\hline V.1 for Embase & 506622 \\
\hline V.3 (Medline) rerun in Embase & 53008 \\
\hline Revised V.3 for Embase with Embase thesaurus & 38347 \\
\hline $\begin{array}{l}\text { Final search } 2 \text { with focused 'population' strand- } \\
\text { remainder of search as before }\end{array}$ & 5262 \\
\hline \multicolumn{2}{|l|}{ Duplicates: $\mathrm{N}=458$} \\
\hline Ready for title and abstract screening & 4804 \\
\hline \multicolumn{2}{|l|}{ Final Embase Search } \\
\hline $\begin{array}{l}\text { Focused 'safety' (search 1)+focused 'population' (search } \\
\text { 2) }=1492+5262\end{array}$ & 6754 \\
\hline \multicolumn{2}{|l|}{ Duplicates removed: $\mathrm{N}=833(=375+458)$} \\
\hline Ready for title and abstract screening $=1117+4804$ & 5921 \\
\hline
\end{tabular}

mixed-methods research for patient safety and palliative care (see figure 2). This was developed and tested via a scoping exercise in the 2003-2015 (approximately 14 million reports) NRLS dataset in order to establish the most effective combination of search terms, balancing rigour, robustness, sensitivity and specificity.

Initially we ran a test search (PALLCARE GENERAL) using the pre-existing codes routinely applied within the NRLS database of (specialty=palliative care) AND (incident=medication). This identified 28000 specialty incidents (A, figure 2) and 75114 medication incidents (B, figure 2) with an overlap of 4435 incidents (C, figure 2).

We then ran a systematically designed search (PALLMED SPECIFIC) using free-text keyword variations in conceptual search strands (see table 5 for a summary of these, the full SQL codes will be made available on completion of the study) to link:

[[Keyword search: drug categories and individual drug names] OR [Keyword search: symptoms, medication delivery route or purpose]] AND [Population: Palliative Phase of illness]

In doing so, we established that relying on standardised categorisations would result in significant underestimating of incident reports as only 449 (J, figure 2) of the resultant hits were also contained in PALLCARE GENERAL (specialty=palliative AND incident $=$ medication $)$.

Our PALLMED SPECIFIC search identified 28091 (H, figure 2) incidents of which 26926 were not formally coded as specialty=palliative and 22023 were not formally coded as incident $=$ medication. Despite this, review of the first 1000 of each of these non-matched sets demonstrated 
Table 4 A researcher-derived strength score descriptors adapted for use in quality assessment for secondary analysis

\begin{tabular}{lll} 
Strength score & $\begin{array}{l}\text { Adapted score descriptors used for secondary } \\
\text { analysis }\end{array}$ & Outcome \\
\hline S1 & $\begin{array}{l}\text { No clear methods leading to results and } \\
\text { conclusions: not significant }\end{array}$ & $\begin{array}{l}\text { Summary description to be included in the } \\
\text { results only and flagged as low quality }\end{array}$ \\
S2 & $\begin{array}{l}\text { Methods lack detail, although results may suggest } \\
\text { a trend (eg, article covers something unique) }\end{array}$ & $\begin{array}{l}\text { Include } \\
\text { S3 }\end{array}$ \\
Methods appropriate for our research question \\
S4
\end{tabular}

\section{Box 2 Scoping review strategy-search steps, ${ }^{1}$ inclusion and exclusion criteria}

1. Initial search of two databases relevant to the topic: Ovid Medline \& Ovid Embase. The background preliminary work to develop our initial limited search in Medline and Embase has been completed and we are now in a position to re-run this search and screen.

2. Analysis of text words in titles and abstracts plus index terms (to produce refined keywords/MeSH terms) of included records.

3. All keywords/MeSH terms of relevance identified run across multiple databases (Ovid Medline, Ovid Embase, Ovid Emcare, Scopus, CINAHL Plus, PsycINFO) and evidence repositories (PROSPERO, Cochrane, NICE Evidence). At this point, search alerts will be set up to ensure any new publications during the course of our study will be identified.

4. Review of reference lists of hits plus forward citation searching.

5. Author contact for clarifications/additional publications if required (eg, to follow-up publications of protocols).

6. Additional grey literature searches: Healthtalk online, UK Palliative Care Organisations (listed at http://www.ncpc.org.uk/uk-palliative-care-organisations), NHS (England, Wales, Scotland and Northern Ireland), International and UK Palliative Care conference abstracts (EAPC, HUK, population, concept, context), plus consideration of searches in other organisations cited in identified literature. Inclusion criteria

- Empirical studies, with quantitative, qualitative or mixed-methods data directly from the population of interest.

- Opinion pieces, editorials, commentaries, letters and other nonempirical academic publications which report first-person experience from the population of interest.

- Focus of item is an aspect of medication management or patient safety in the context of palliative care.

- No restriction by location of patient.

- English language.

- No date of publication restrictions or publication status restrictions.

No restriction by country of origin for study or researchers.

Exclusion criteria

- Not a first-person account or no first-person data included.

- Account from the perspective of healthcare professional caregivers or social care professional caregivers only.

- Paid agency carer accounts.

Does not meet concept definitions. that these reports were still within scope. Furthermore, in PALLCARE GENERAL there were 3986 hits (D, figure 2) that were not identified in PALLMED SPECIFIC.

An additional 716 hits (I, figure 2) shared between specialty=palliative (but not incident=medication) and PALLMED SPECIFIC and 6068 (K, figure 2) shared between incident=medication (but not specialty=palliative) and PALLMED SPECIFIC. Therefore, overall

\section{Box 3 Additions/removals in Medline and Embase with reasons for changes}

Terms added: medicine* and secret*

Terms removed: carers, volunteers, interpersonal relations, significan* patient, others significan*, relative, general public, patient*, inpatient*, care $^{\star}$, care? giver*, family, care giver ${ }^{*}$, care-giver ${ }^{\star}$, caregiver*, families, parent?, friend?, relative?, spouse?, partner?, husband?, wife, wives, child, children, close person?, significant other.

Reasons

a. If searched as a MESH term, then not used as a keyword (once duplication confirmed) for example, patient* removed because covered by patient as a MESH term

b. Volunteers and general public were removed because not connected to patient/not specific enough.

c. Interpersonal relations, quality of healthcare, drug*, analgesic, adjuvant $^{*}$, antisecret* ${ }^{*}$ secretion, bereave* ${ }^{\star}$, attitude to death-removed as not specific enough to be useful.

d. significan*/relative/families/advocate/unsafe/harm/adverse event/ hospice ${ }^{\star}$, palliative*, advanced, morphine AND cancer, cancer pain were all tested as exploded terms. This did not add any relevant records to the results beyond other selected terms and so these were removed.

In Embase removal of the following terms were made during testing as these were not providing any additional relevant records: inpatient*, care $^{\star}$, care? giver ${ }^{\star}$, care giver ${ }^{\star}$, care-giver* ${ }^{\star}$, caregiver*, parent?, friend, spouse?, partner?, husband?, wife, wives, child, children, close person?, significant other, attitude to death. In addition, 'Macmillan' keyword was removed since its yield was 34188 of which most were included if Macmillan Publishers Ltd related and 'ACP' as a keyword was removed as it yielded 10590 and it is an abbreviation for many terms; advanced care planning as a SH term continued to be included and has been exploded. 


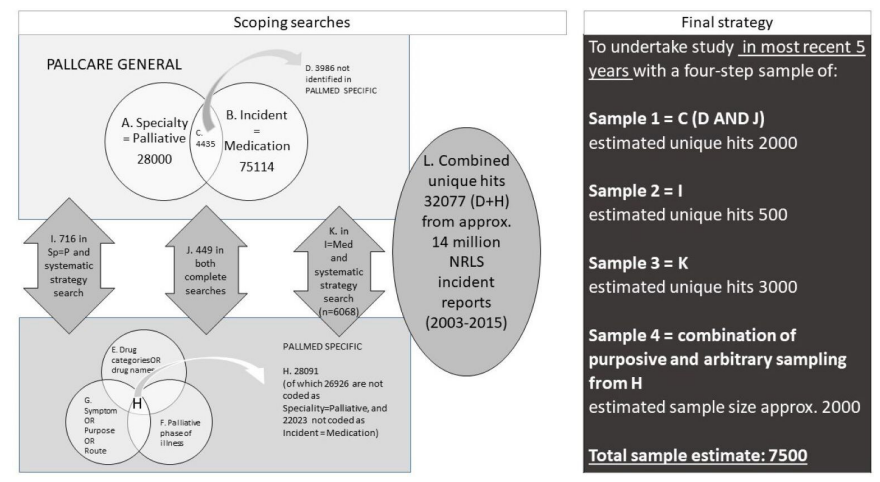

Figure 2 Database scoping and search design.

hits from all our searches of potentially relevant reports $=28091+3986=32077$ (L, figure 2)

We suspect the findings of our scoping exercise are largely because palliative care is a complex and poorly defined field with a long history of debate surrounding what 'counts' as palliative care, when and where this should be delivered (home or usual place of residence, hospital, hospice), which aspects of care should be delivered by who (specialists or generalists), and if it should be defined by time frame (days, weeks, months or years) or intention (end-of-life care, palliative or curative, symptom control or disease modifying). Clearly this presents challenges for trained coders, and even more so for front-line clinical staff when entering data into local systems that then map to the NRLS.

The right-hand box of figure 2 'Final strategy' shows the further detail of our sampling strategy for the study. We will initially draw three samples designed to ensure rigour and sensible use of resource by balancing sensitivity with specificity focusing on the reports most likely to provide rich data. To include all of $\mathrm{L}$, figure 2 would be sensitive but unmanageable. We will therefore use $\mathrm{C}$ (encompassing D and J), followed by I and then K, before sampling further from $\mathrm{H}$. The details of the purposive part of this sampling will be developed once samples 1-3 are analysed to ensure sample 4 is designed to capture a combination of the richest data, providing depth and breadth to the study sample. A total of 1000 arbitrarily selected reports from within $\mathrm{H}$ will also be used.

The search strategy will initially be applied to the most recent 60 months from the current NRLS database. With the above strategy we estimate this will produce approximately 7500 reports to analyse. If this results in less than 7500 individual incidents it will be extended back year by year to reach this target sample size which is based on our prior knowledge of reporting frequency and trends.

\section{Data cleaning}

As with many large routinely generated datasets, data cleaning will be required to ensure the final dataset only contains incidents pertaining to the study aim and objectives. NRLS data entry by front-line healthcare professionals is already reviewed centrally to anonymise it, removing specific names of people, places and organisations. Our cleaning process (at least 2 members of the research team, with a third in cases of uncertainty) will ensure study inclusion and exclusion criteria are met. These are given in table 6 and have been previously used successfully to select palliative care incidents in similarly constructed databases. ${ }^{3}$

Once a clean dataset is obtained new unique identifiers (by setting) will be allocated to each incident report to ensure anonymity alongside traceability during the analytical process.

\section{Analysis}

The extracted sample of reports will be reviewed and accurately reclassified (coded) using the PISA classification system for the purposes of descriptive quantification.

The PISA classification system has been used in over 30 health service research studies and is aligned with key concepts for understanding patient safety described in the WHO International Classification for Patient Safety ${ }^{53} 65$ The system is comprised of multiaxial frameworks developed using a constant comparative methods to describe incidents, contributing factors (circumstances, actions or influences perceived to play a part in the origin or development of an incident), type and severity of harm. It has been successfully applied to other areas of palliative care. ${ }^{2766}$ PISA will be used to quantitatively characterise (context, setting, patient-related features, severity of harm) the data, identifying frequencies of occurrence and characteristics of incident thematic groupings (by setting, task etc).

A modified framework analysis ${ }^{67-69}$ will be used to qualitatively analyse free-text within the data, in order to interpret how the reporters perceived each incident, including the meanings attributed to it, and develop depth of understanding regarding how and why incidents might occur (including underlying sociocultural factors). ${ }^{70}$ Attention will be paid to language, metaphors and indicators of interaction between people and artefacts within the complexities of the healthcare systems delivering palliative care across different settings (hospitals, hospices, community) and providers (specialist and generalist services). This analysis will be used to build a richer picture of professional conceptualisations of what is worthy of reporting and hence what constitutes unsafe care, contributing factors, when blame (directed to self or others) ${ }^{71}$ or other value judgements are attributed to systems or human error.

We will document if any part of the analysis has not been possible due to missing information. We know from previous studies that it is likely approximately $15 \%-20 \%$ of reports will not contain free text data for qualitative analysis. ${ }^{27}$ If the available reports containing suitable free text data exceed 2000, we will purposively sample within our dataset for the qualitative work, drawing on the quantitative findings to do so. While we have taken great care to produce a representative sample of medication-related patient safety incidents it remains possible that some specific elements may be under or over-represented due 


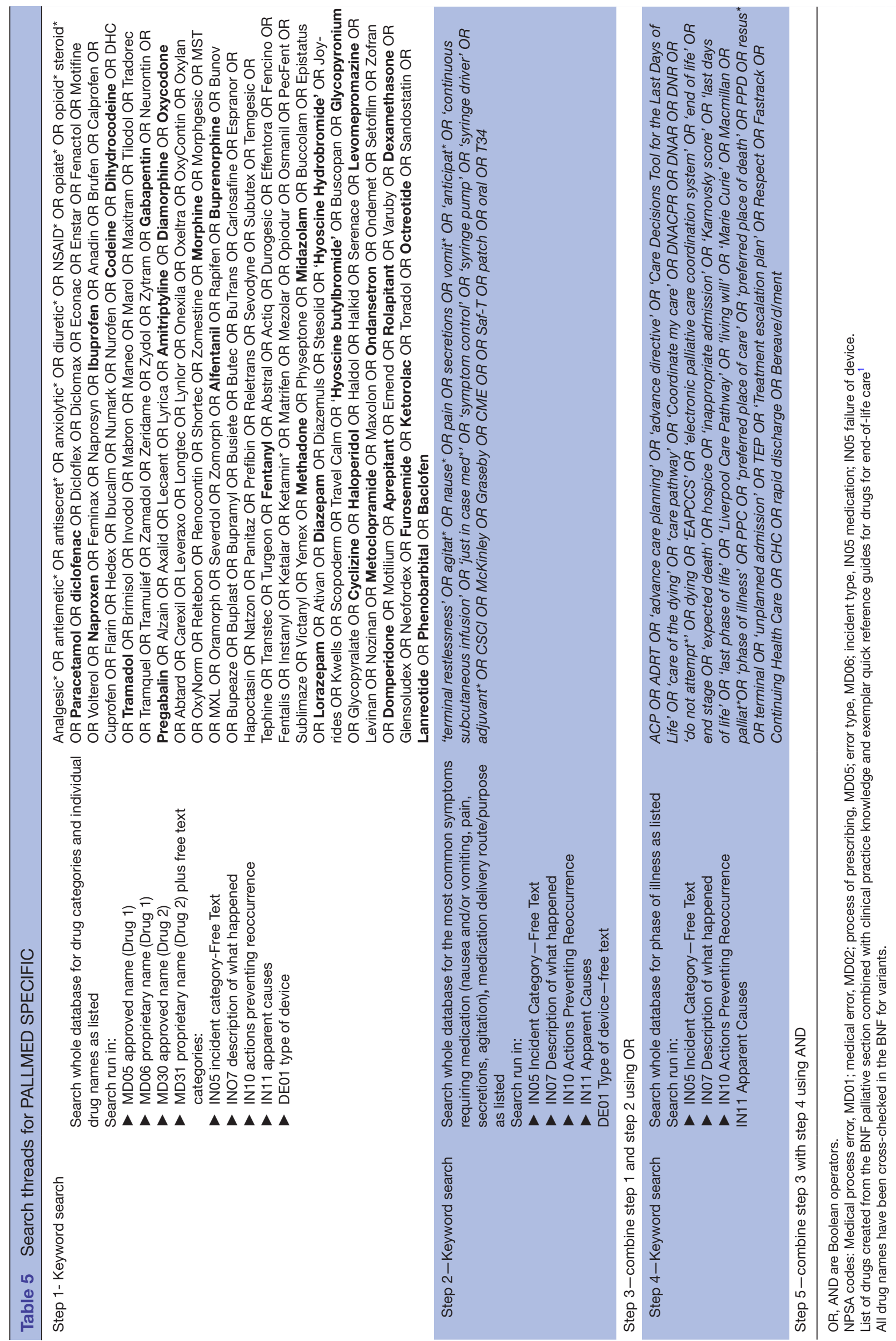


Table 6 Three stages of data extraction for analysis

1. Codes and associated free text data to be extracted from the NRLS database

Unique anonymised incident ID Numerical

\begin{tabular}{ll}
\hline Date of incident & Date \\
\hline Incident type & Structured \\
\hline Degree of harm (severity) & Structured \\
\hline Incident location & Structured \\
\hline Specialty data/professions involved & Structured \\
\hline Description of what happened & Unstructured/free text \\
\hline Actions preventing reoccurrence & Unstructured/free text \\
\hline Apparent causes & Unstructured/free text \\
\hline
\end{tabular}

Apparent causes

Unstructured/free text

2. Categorisation using the Primary care patient safety classification (PISA) coding framework

Initial screening (with $20 \%$ checked by a second independent coder) before decision include/exclude on study scope.

- Reason for exclusion or final decision for quantitative analysis include/ exclude.

Exclusions

*Setting of occurrence will be coded as: hospice/acute hospital/usual place of residence (own home)/usual place of residence (residential care)/usual

place of residence (nursing care)/general practice surgery/other institutional setting/other non-institutional setting (home of relative/friend/informal carer) unknown/other

- Patient not in last phase of life (defined as having potentially life-limiting irreversible or progressive condition requiring general or specialist

Medication process will be coded as: Decision making/starting a medication. care for symptom control, social, psychological and/or spiritual Prescribing/taking/adding a medication. support).

- Not a medication process.

- Medications used without palliative intent (eg, for anaesthetic procedures, incident solely related to disease-modifying treatment for example, chemotherapy drug errors).

Monitoring and supply/reviewing a medication.

- Administration.

- Stopping medications.

- Moving across healthcare contexts.

- Other.

- Incident not related to patient care.

Inclusions

1. Incident report includes any of the following:Care provided in a hospice inpatient unit.

2. Care provided by a specialist palliative medicine team.

A clear statement of a decision to treat with palliative (as opposed to

life-prolonging) intent prior to the incident occurring in any other setting. If included:

PISA incident types

PISA contributing factors

- PISA outcomes

- Setting of Occurrence*

- Informal carers involved

- Drugs involved

- Medication process - point of error or risk ${ }^{* *}$

- Harm outcome

- Harm severity physical

- Harm severity emotional/psychological

- Coder notes

3. Inclusion for qualitative analysis (yes/no-only if insufficient free text)

Interpretative analysis including:

- Use of language, metaphors, the reporters' stance and construction of the incident.

Who is reporting what, when, why and for what purpose.

NRLS, National Reporting and Learning System.

to the nature of the primary data being dependent on what people choose to report. We will account for major changes in practice (eg, withdrawal of the Liverpool Care Pathway, COVID-19 pandemic impact) in the analysis by reviewing incidents with attention to the chronological timeline of reporting. We also recognise that social care providers and private companies will not be represented unless NHS organisations (services or staff) are also involved. To investigate this, issue will require another study.

\section{Integration of study components}

Given the data diversity, we will use narrative methods to integrate findings of the scoping review with those from analysis of NRLS reports. This will compare and contrast what front-line healthcare professionals have chosen to report, and why, with known patient and informal carer views on what is important. We will also explore the range of potential impact of both rare and common types of incidents in a holistic sense by drawing on both study components. We will use this to develop a conceptual model 
(representing the integrated findings) for meaningful learning from incidents that integrate patient and carer perspectives, to inform professional training to enhance safety and quality. We anticipate drawing on conceptual models for whole system and human factor issues within the wider field of patient safety to achieve this.

\section{Ethics and dissemination}

\section{Ethical approval and study governance}

Prospective ethical approval for the study has been granted from Cardiff University School of Medicine Research Ethics Committee (Ref 19/28). A data sharing agreement exists between Cardiff University and NHS Improvement. Data released to the research team will be anonymised based on in-house data cleaning processes led by the NHS Improvement team. Should the research team recognise identifiable content in a report, we will follow strict Information Governance procedures to notify NHS Improvement.

\section{Anticipated outcomes}

This work will provide evidence for meaningful patient safety initiatives in palliative medication management. ${ }^{72}$ Our study will, for the first time, bring together multivoiced constructions of patient safety in palliative care in order to identify the implications for professional learning and actions. These lessons are relevant across health and social care, particularly in the context of an ageing multimorbid population increasingly in need of palliation alongside disease-modifying interventions. New knowledge of how patients and professionals construct ideas of what constitutes (un)safe care from this study will underpin targeted quality improvement projects and prioritisation of further research. For example, identifying the most frequent or highest impact incidents, and their underlying contributory factors, provides signals to pinpoint where and how healthcare services and teams can focus resources to design safer systems for patients.

Generation of the dataset using methods originating from best practice for systematic reviews is a novel methodological approach. The methodological developments will be transferable and contribute to the evidence base for safety work in palliative care and other clinical areas.

\section{Dissemination}

The study team includes academic clinicians (doctors and a pharmacist) who have access through their professional networks to share emerging findings with clinical/professional groups through internal/external seminars and conference presentations. Open-access dissemination events (according to pandemic restrictions) will be held at the end of the study in collaboration with our NHS funding partner and with advertising to NHS and charitable providers of specialist/generalist palliative care and the public including patients and informal carers. We will continually monitor and record the impact of our PPI involvement methods throughout the study processes so that we can evaluate and further learn from it. The PPI collaborator and stakeholder dissemination events are funded. ${ }^{73}$

We anticipate publication of the final findings within 12 months of completion of the study, which we will complete within 12 months of receiving the complete NRLS sample dataset for the study.

The study team will submit the results to high-impact peer-reviewed journals and undertake national and international oral presentations to researchers, clinicians and policymakers. Throughout, the research updates will be shared via press releases and social media. Plain English summaries will be prepared for the general public. We will work with NHS England to achieve this.

The findings of this research will be directly relevant to front-line clinical practice and professional learning, with implications for prevention and mitigation of patient safety incidents. Findings will help inform targeted education and quality improvement initiatives by providing evidence for synergies and dissonance between patients, informal carers and professionals' views of what makes (un)safe care. The work will also inform methods in practice for coding and reporting patient safety in palliative care.

\section{Author affiliations}

${ }^{1}$ Marie Curie Palliative Care Research Department, University College London, London, UK

${ }^{2}$ Central and North West London NHS Foundation Trust, London, UK

${ }^{3}$ Patient and Public Involvement Collaborator, Hayward Heaths, UK

${ }^{4}$ Division of Population Medicine, School of Medicine, Cardiff University, Cardiff, UK

${ }^{5}$ Patient Safety Team, NHS England and NHS Improvement, London, UK

\section{Twitter Sally-Anne Francis @sally_anne_fran}

Acknowledgements The systematic scoping review search strategy was kindly peer-reviewed by Mala K Mann Information Specialist/Systematic Reviewer, SURE University Library Service, Cardiff University. We thank Frances Healey for her constructive comments on an earlier version of this protocol, and also for her work, together with the wider NHS Improvement Team in collaborating with us in extracting the data required from the NRLS database.

Collaborators The systematic scoping review search strategy was kindly peerreviewed by Mala K Mann Information Specialist/Systematic Reviewer, SURE University Library Service, Cardiff University.We thank Frances Healey for her constructive comments on an earlier version of this protocol, and also for her work, together with the wider NHS Improvement Team in collaborating with us in extracting the data required from the NRLS database.

Contributors SY conceived the idea for the study and developed the full protocol from the initial stages to final version in close collaboration with AC-S and AC. AC is our PPI collaborator. He is an expert by experience in both providing PPI input to a significant number of research projects within patient safety and the wider NHS research remit. S-AF and SY created and tested the systematic search strategy for the scoping review. SH and SY developed the systematic search for the NRLS database which was then refined in collaboration with JA. All authors contributed to the writing of this manuscript.

Funding This work is supported by the Central and North West London NHS Foundation Trust Starter Grant Scheme.

Competing interests None declared.

Patient consent for publication Not required.

Provenance and peer review Not commissioned; externally peer reviewed.

Supplemental material This content has been supplied by the author(s). It has not been vetted by BMJ Publishing Group Limited (BMJ) and may not have been peer-reviewed. Any opinions or recommendations discussed are solely those of the author(s) and are not endorsed by BMJ. BMJ disclaims all liability and 
responsibility arising from any reliance placed on the content. Where the content includes any translated material, BMJ does not warrant the accuracy and reliability of the translations (including but not limited to local regulations, clinical guidelines, terminology, drug names and drug dosages), and is not responsible for any error and/or omissions arising from translation and adaptation or otherwise.

Open access This is an open access article distributed in accordance with the Creative Commons Attribution Non Commercial (CC BY-NC 4.0) license, which permits others to distribute, remix, adapt, build upon this work non-commercially, and license their derivative works on different terms, provided the original work is properly cited, appropriate credit is given, any changes made indicated, and the use is non-commercial. See: http://creativecommons.org/licenses/by-nc/4.0/.

\section{ORCID iDs}

Sarah Yardley http://orcid.org/0000-0002-1645-642X

Sally-Anne Francis http://orcid.org/0000-0003-2384-1518

\section{REFERENCES}

1 World Health Organisation. Medication without harm: WHO's third global patient safety challenge, 2017. Available: http://www.who.int/ patientsafety/medication-safety/en/ [Accessed 12 Mar 2018].

2 NHS England. Patient safety incident reporting continues to improve, 2020. Available: https://improvement.nhs.uk/resources/nationalpatient-safety-incident-reports-25-march-2020/ [Accessed 27 Jul 2020].

3 Yardley I, Yardley S, Williams $\mathrm{H}$, et al. Patient safety in palliative care: a mixed-methods study of reports to a national database of serious incidents. Palliat Med 2018;32:1353-62.

4 Smith J. Building a safer NHS for patients: improving medication safety. London: Department of Health, 2004. http://webarchive. nationalarchives.gov.uk/20130107105354/http://www.dh.gov.uk/ prod_consum_dh/groups/dh_digitalassets/@dh/@en/documents/ digitalasset/dh_4084961.pdf

5 Lewis PJ, Dornan T, Taylor D, et al. Prevalence, incidence and nature of prescribing errors in hospital inpatients: a systematic review. Drug Saf 2009;32:379-89.

6 Ross S, Hamilton L, Ryan C, et al. Who makes prescribing decisions in hospital inpatients? an observational study. Postgrad Med J 2012;88:507-10.

7 Ross S, Ryan C, Duncan EM, et al. Perceived causes of prescribing errors by junior doctors in hospital inpatients: a study from the protect programme. BMJ Qual Saf 2013;22:97-102.

8 Tully MP, Ashcroft DM, Dornan T, et al. The causes of and factors associated with prescribing errors in hospital inpatients: a systematic review. Drug Saf 2009;32:819-36.

9 Brennan N, Mattick K. A systematic review of educational interventions to change behaviour of prescribers in hospital settings, with a particular emphasis on new prescribers. Br J Clin Pharmacol 2013;75:359-72.

10 Dean B, Schachter M, Vincent C, et al. Causes of prescribing errors in hospital inpatients: a prospective study. Lancet 2002;359:1373-8.

11 Woodward HI, Mytton OT, Lemer C, et al. What have we learned about interventions to reduce medical errors? Annu Rev Public Health 2010;31:479-97.

12 Mazzocato C, Stiefel F. How safe are opioids in palliative care? Support Care Cancer 1997;5:427.

13 Shaheen PE, Legrand SB, Walsh D, et al. Errors in opioid prescribing: a prospective survey in cancer pain. J Pain Symptom Manage 2010;39:702-11.

14 McCormack PJ, Cooper R, Sutherland S, et al. The safe use of syringe drivers for palliative care: an action research project. Int $J$ Palliat Nurs 2001;7:574-80.

15 O'Brien H, Kiely F, Carmichael A. Doctor-related medication safety incidents on a specialist palliative medicine inpatient unit: a retrospective analysis of three years of voluntary reporting. $J$ Pain Palliat Care Pharmacother 2017;31:105-12.

16 Casarett D, Spence C, Clark MA, et al. Defining patient safety in hospice: principles to guide measurement and public reporting. $J$ Palliat Med 2012;15:1120-3.

17 Smucker DR, Regan S, Elder NC, et al. Patient safety incidents in home hospice care: the experiences of hospice interdisciplinary team members. J Palliat Med 2014;17:540-4.

18 Dietz I, Borasio GD, Molnar C, et al. Errors in palliative care: kinds, causes, and consequences: a pilot survey of experiences and attitudes of palliative care professionals. J Palliat Med 2013;16:74-81.

19 Dietz I, Plog A, Jox RJ, et al. "Please describe from your point of view a typical case of an error in palliative care": Qualitative data from an exploratory cross-sectional survey study among palliative care professionals. J Palliat Med 2014;17:331-7.

20 Dietz I, Borasio GD, Schneider G, et al. Medical errors and patient safety in palliative care: a review of current literature. J Palliat Med 2010;13:1469-74.

21 Heneka N, Shaw T, Rowett D, et al. Quantifying the burden of opioid medication errors in adult oncology and palliative care settings: a systematic review. Palliat Med 2016;30:520-32.

22 World Health Organization. Conceptual framework for the International classification for patient safety version 1.1, 2009. Available: https://www.who.int/patientsafety/taxonomy/icps_full_ report.pdf [Accessed 4 Aug 2020].

23 Kajamaa A, Mattick K, Parker H, et al. Trainee doctors' experiences of common problems in the antibiotic prescribing process: an activity theory analysis of narrative data from UK hospitals. BMJ Open 2019;9:e028733.

24 Bowers B, Ryan R, Kuhn I, et al. Anticipatory prescribing of injectable medications for adults at the end of life in the community: a systematic literature review and narrative synthesis. Palliat Med 2019;33:160-77

25 Dy SM. Patient safety and end-of-life care: common issues, perspectives, and strategies for improving care. Am J Hosp Palliat Care 2016;33:791-6.

26 NHS Improvement. Patient safety. Available: https://improvement. nhs.uk/improvement-hub/patient-safety/\#: :text=Patient\%20safety-, Patient\%20safety,improvements\%20in\%20safety\%20and\% 20quality [Accessed 27 Aug 2020].

27 Williams $\mathrm{H}$, Noble S, Edwards A. Designing safer services for patients needing palliative care in GP out of hours services: a national analysis of patient safety data to identify priorities for systems improvement. BMJ Support \& Palliat Care 2018;8:A10.

28 National Institute of Clinical Excellence. Palliative care for adults: strong opioids for pain relief. London: NICE, 2012. https://www.nice. org.uk/guidance/cg140

29 Carduff E, Johnston S, Winstanley C, et al. What does 'complex' mean in palliative care? triangulating qualitative findings from 3 settings. BMC Palliat Care 2018;17:12.

30 Ryan-Woolley B, McHugh G, Luker K. Exploring the views of nurse prescribing among macmillan nurses. $\mathrm{Br} J$ Community Nurs 2008;13:171-7.

31 den Herder-van der Eerden M, van Wijngaarden J, Payne S, et al. Integrated palliative care is about professional networking rather than standardisation of care: a qualitative study with healthcare professionals in 19 integrated palliative care initiatives in five European countries. Palliat Med 2018;32:1091-102.

32 NHS England. What is NHS rightcare? Available: https://www. england.nhs.uk/rightcare/what-is-nhs-rightcare/ [Accessed 27 Aug 2020].

33 Leadership Alliance for the Care of Dying People. One chance to get it right: Improving people's experience of care in the last few days and hours of life. London: LACDP, 2014. https://assets. publishing. service.gov.uk/government/uploads/system/uploads/attachment_ data/file/323188/One_chance_to_get_it_right.pdf

34 Collier A, Sorensen R, ledema R. Patients' and families' perspectives of patient safety at the end of life: a video-reflexive ethnography study. Int J Qual Health Care 2016;28:66-73.

35 Kiesewetter I, Schulz C, Bausewein C, et al. Patients' perception of types of errors in palliative care - results from a qualitative interview study. BMC Palliat Care 2016;15:75.

36 Pound P, Britten N, Morgan M, et al. Resisting medicines: a synthesis of qualitative studies of medicine taking. Soc Sci Med 2005;61:133-55.

37 Etkind SN, Bone AE, Lovell N, et al. The role and response of palliative care and hospice services in epidemics and pandemics: a rapid review to inform practice during the COVID-19 pandemic. $J$ Pain Symptom Manage 2020;60:e31-40.

38 Antunes B, Bowers B, Winterburn I, et al. Anticipatory prescribing in community end-of-life care in the UK and ireland during the COVID-19 pandemic: online survey. BMJ Support Palliat Care 2020;10:343-9.

39 Bone AE, Finucane AM, Leniz J, et al. Changing patterns of mortality during the COVID-19 pandemic: population-based modelling to understand palliative care implications. Palliat Med 2020;34:1193-201.

40 Bowers B, Pollock K, Lovick R. Unintended consequences of rapid changes in community palliative care practice, 2020. BJGP Life. Available: https://bjgplife.com/2020/11/02/unintendedconsequences-of-rapid-changes-in-community-palliative-carepractice/

41 Lam JKW, Cheung CCK, Chow MYT, et al. Transmucosal drug administration as an alternative route in palliative and end-of- 
life care during the COVID-19 pandemic. Adv Drug Deliv Rev 2020;160:234-43.

42 Palliative and end of life care Priority Setting Partnership (PeolcPSP) Putting patients, carers and clinicians at the heart of palliative and end of life care research. London: James Lind Alliance, 2015. https:// www.jla.nihr.ac.uk/priority-setting-partnerships/palliative-and-end-oflife-care/downloads/Palliative-and-end-of-life-care-PSP-final-report. pdf

43 Public Involvement Standards Development Partnership. UK standards for public involvement. National standard 2: working together. Available: https://sites.google.com/nihr.ac.uk/pi-standards/ home [Accessed 17 Jun 2019].

44 INVOLVE. Guidance on co-producing a research project. London: NIHR, 2018. http://www.invo.org.uk/wpcontent/uploads/2018/03/ Copro Guidance Mar18.pdf

45 Public Involvement Standards Development Partnership. UK standards for public involvement. National standard 6: governance. Available: https://sites.google.com/nihr.ac.uk/pi-standards/home [Accessed 17 Jun 2019].

46 Public Involvement Standards Development Partnership. UK standards for public involvement. National standard 5: impact. Available: https://sites.google.com/nihr.ac.uk/pi-standards/home [Accessed 17 Jun 2019].

47 Public Involvement Standards Development Partnership. UK standards for public involvement. National standard 4: communications. Available: https://sites.google.com/nihr.ac.uk/pistandards/home [Accessed 17 Jun 2019].

48 Public Involvement Standards Development Partnership. Nationa standard 1: inclusive opportunities. Available: https://sites.google. com/nihr.ac.uk/pi-standards/home [Accessed 17 Jun 2019].

49 Blumer H. Symbolic interactionism: perspective and method. Berkeley: University of California Press, 1969.

50 Crotty M. Constructionism: the making of meaning. In: The foundations of social research: meaning and perspective in the research process. London: Sage Publications, 1998. https://uk.sagep ub.com/en-gb/eur/the-found ation s-of-socia l-resea rch/book2 07972

51 Denzin NK. Interpretive interactionism. 2nd edn. Thousand Oaks: Sage Publications, 2001.

52 Carson-Stevens A, Hibbert P, Williams H. Characterising the nature of primary care patient safety incident reports in the England and Wales national reporting and learning system: a mixed-methods agendasetting study for general practice. Southampton, UK: NIHR Journals Library, 2016.

53 Carson-Stevens A, Hibbert P, Avery A, et al. A cross-sectional mixed methods study protocol to generate learning from patient safety incidents reported from general practice. BMJ Open 2015;5:e009079.

54 Colquhoun HL, Levac D, O'Brien KK, et al. Scoping reviews: time for clarity in definition, methods, and reporting. J Clin Epidemiol 2014;67:1291-4.

55 Arksey H, O'Malley L. Scoping studies: towards a methodological framework. Int J Soc Res Methodol 2005;8:19-32.

56 Decaria JE, Sharp C, Petrella RJ. Scoping review report: obesity in older adults. Int $J$ Obes 2012;36:1141-50.

57 The Joanna Briggs Institute. The joanna briggs institute reviewers' manual 2015: methodology for jbi scoping reviews. South Australia:
The University of Adelaide, 2015. https://nursing.Isuhsc.edu/JBI/ docs/ReviewersManuals/Scoping-.pdf

58 Cottrell E, Yardley S. Lived experiences of multimorbidity: an interpretative meta-synthesis of patients', general practitioners' and trainees' perceptions. Chronic IIIn 2015;11:279-303.

59 Noblit GW, Hare RD. Meta-ethnography: synthesizing qualitative studies. California: Sage, 1988.

60 Tong A, Flemming K, Mclnnes E, et al. Enhancing transparency in reporting the synthesis of qualitative research: ENTREQ. BMC Med Res Methodol 2012;12:181.

61 Brooks D, Fielding E, Beattie E, et al. Effectiveness of psychosocial interventions on the psychological health and emotional wellbeing of family carers of people with dementia following residential care placement: a systematic review. JBI Database System Rev Implement Rep 2018;16:1240-68.

62 Gough D. Weight of evidence: a framework for the appraisal of the quality and relevance of evidence. Res Pap Educ 2007;22:213-28.

63 Enhancing the quality and transparency of health research. Available: https://www.equator-network.org/

64 NHS England. Everyone over 16 can now get their COVID-19 jab, 2021. Available: https://improvement.nhs.uk/documents/2549/ NRLS Guidance notes March 2018.pdf

65 Carson-Stevens A, Hibbert P, Williams H, et al. Characterising the nature of primary care patient safety incident reports in the England and Wales national reporting and learning system: a mixed-methods agenda-setting study for general practice. Health Serv Deliv Res 2016;4:1-76.

66 Dinnen T, Williams H, Yardley S, et al. Patient safety incidents in advance care planning for serious illness: a mixed-methods analysis. BMJ Support Palliat Care 2019. doi:10.1136/ bmjspcare-2019-001824. [Epub ahead of print: 28 Aug 2019].

67 Crotty M. The foundations of social research: meaning and perspective in the research process. London: SAGE, 1998.

68 Gale NK, Heath G, Cameron E, et al. Using the framework method for the analysis of qualitative data in multi-disciplinary health research. BMC Med Res Methodol 2013;13:117.

69 Pope C. Analysing qualitative data. BMJ2000;320:114. doi:10.1136/ bmj.320.7227.114

70 Smith JA. Beyond the divide between cognition and discourse: using interpretative phenomenological analysis in health psychology. Psychol Health 1996;11:261-71.

71 Cooper J, Edwards A, Williams $\mathrm{H}$, et al. Nature of blame in patient safety incident reports: mixed methods analysis of a national database. Ann Fam Med 2017;15:455-61.

72 Vincent CA. Analysis of clinical incidents: a window on the system not a search for root causes. Qual Saf Health Care 2004;13:242-3.

73 Public Involvement Standards Development Partnership. National standard 3: support and learning. Available: https://sites.google.com/ nihr.ac.uk/pi-standards/home [Accessed 17 Jun 2019].

74 Ryan R, Bowers B, Spathis A. 11 how is anticipatory prescribing in community end of life care governed in the UK? a content analysis of national and local guidance documents. BMJ Supportive \& Palliative Care 2020;10:A4-5.

75 WHO. 5 moments for medication safety, 2019. Available: https:// apps.who.int/iris/bitstream/handle/10665/311155/WHO-HIS-SDS2019.6-eng.pdf?ua=1 [Accessed 21 Dec 2020]. 